\title{
Avaliação da atividade da mieloperoxidase neutrofílica em bovinos da raça Holandesa e sua correlação com níveis plasmáticos de ácido ascórbico
}

\section{Evaluation of neutrophilic myeloperoxidase activity in Holstein cows and its correlation to plasma ascorbic acid levels}

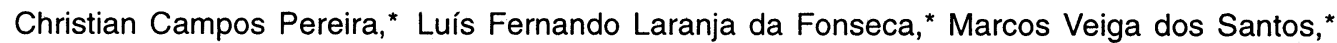 \\ Paulo Henrique Mazza Rodrigues, ${ }^{*}$ Primavera Borelli**
}

\begin{abstract}
Resumo
A mieloperoxidase (MPO) é uma enzima que desempenha papel fundamental nos mecanismos bactericidas dos mamíferos, sendo encontrada principalmente em leucócitos polimorfonucleares neutrófilos. A MPO está diretamente relacionada com os mecanismos bactericidas dependentes de $\mathrm{O}_{2}$, sendo catalisadora do processo de formação de radicais altamente reativos como o ácido hipocloroso (HCLO), a partir de $\mathrm{H}_{2} \mathrm{O}_{2}$ e íons halogênios, constituindo, portanto, importante mecanismo de defesa contra microrganismos patogênicos. O ácido ascórbico apresenta efeitos estimulatórios sobre a atividade fagocitária de leucócitos, sobre a função do sistema reticuloendotelial e sobre a síntese de anticorpos, além de estimular a atividade de MPO in vitro em humanos, aumentando a produção de $\mathrm{HClO}^{-}$. No homem, foi descrito um quadro de deficiência de MPO que leva à diminuição da capacidade bactericida dos neutrófilos contra Staphylococcus aureus e Candida albicans, e também a uma maior susceptibilidade desses indivíduos às infecções em geral. Neste trabalho foram coletadas amostras de sangue de 100 vacas da raça Holandesa, distribuídas em diferentes estágios de lactação e provenientes de três rebanhos comerciais. Utilizou-se o método de Kaplow, originalmente utilizado em humanos, para a avaliação da atividade MPO de neutrófilos e a técnica de HPLC ("High Performance Liquid Chromatograph") para a determinação dos níveis plasmáticos de ácido ascórbico. Os resultados da correlação entre a atividade MPO e os níveis de ácido ascórbico foram analisados através do programa "Computacional Statistical Analysis System" (SAS Institute Inc., 1985), mostrando associação negativa entre a atividade enzimática da MPO e o nível plasmático de ácido ascórbico in vivo. Além disto, houve efeito significativo do número de lactações sobre esta associação.
\end{abstract}

Palavras-chave: mieloperoxidase; neutrófilos; ácido ascórbico; bovinos.

\begin{abstract}
The activity of polymorphonuclear leukocytes (PMNL) or neutrophils is critical for the immune response against invading bacteria by phagocytosis and killing. One of the main neutrophil killing mechanisms is the myeloperoxidase-halide system (MPO) that plays a fundamental role in the oxygen-dependent bactericidal mechanism of neutrophil. MPO has a catalytic effect on highly reactive radicals, such as $\mathrm{HOCl}$, when combined with $\mathrm{H}_{2} \mathrm{O}_{2}$ and halide. Ascorbic Acid stimulates leukocyte fagocytic activity, antibody synthesis and MPO activity in humans in vitro. It was collected blood samples from $100 \mathrm{Holstein}$ mature cows from 3 commercial dairy herds of São Paulo state (Brazil) in different stages of lactation. Blood samples were collected from the jugular vein, and blood smears were done in glass slides, as described by Kaplow (1965). Ascorbic Acid was measured by HPLC (High Performance Liquid Chromatograph). There was a negative relationship between MPO activity and plasmatic Ascorbic Acid levels in vivo. Besides that, there was a significant effect of lactation number on that association.
\end{abstract}

Keywords: myeloperoxidase; neutrophil; ascorbic acid; bovine.

\section{Introdução}

A mastite é a doença que mais onera a produção leiteira no mundo. Analisando a prevalência da mastite como doença infecciosa, alguns autores registraram que cerca de $30 \%$ das vacas americanas estão infectadas (Philpot e Nickerson, 1991), sendo que no Brasil, alguns estudos indicam variação de $20 \%$ a $38 \%$ (Fonseca, 1992; Langenegger et al., 1970). Dentre os agentes causadores de mastite destaca-se o Staphylococcus aureus, apontado pela International Dairy

\footnotetext{
* Universidade de São Paulo - Faculdade de Medicina Veterinária e Zootecnia - Departamento de Nutrição e Produção Animal - Pirassununga-SP - Brasil.

** Universidade de São Paulo - Faculdade de Ciências Farmacêuticas - São Paulo, SP - Brasil.

As correspondências poderão ser encaminhadas para: Prof. Dr. Luís Fernando Laranja da Fonseca. Av. Duque de Caxias Norte, 225 - Caixa Postal 23 CEP 13630-000 Pirassununga, SP - Fone: (19) 561-6712 ou (19) 561-6495 ramal 511 - Fax: (19) 561-6215 - E-mail: Ifonseca@ usp.br
} 
Federation (1991) como o principal agente etiológico em 18 de 23 países analisados. No Brasil, vários estudos confirmam a predominância desta bactéria como principal agente causador da mastite bovina (Langenegger et al., 1970; Langoni et al., 1990). A dificuldade de controlar este agente infeccioso está relacionada à baixa eficácia dos tratamentos com antimicrobianos e as reduzidas taxas de cura espontânea, levando a um insatisfatório nível de eliminação das infecções existentes (Sears, 1993). Alguns autores destacam que faIhas nos mecanismos de morte intracelular também podem contribuir para esta baixa taxa de cura espontânea (Cooray e Hakansson, 1995; Santos et al., 1996).

A mieloperoxidase (MPO) é uma hemoproteína lisossomal localizada nos grânulos primários de leucócitos polimorfonucleares (Cooray, 1994; Cooray et al., 1993; Klebanoff, 1991) que na presença de peróxido de hidrogênio $\left(\mathrm{H}_{2} \mathrm{O}_{2}\right)$ e íons halogênios constitui-se em potente sistema bactericida (Klebanoff, 1991), podendo, também, ser efetiva contra fungos, vírus, micoplasmas e células tumorais de mamíferos. Sua ação bactericida ocorre após a ingestão da partícula pelos polimorfonucleares (PMN). Durante o burst oxidativo ocorre a redução do $\mathrm{O}_{2}$ em ânion superóxido que é reduzido a $\mathrm{H}_{2} \mathrm{O}_{2}$. A MPO tem ação catalítica na formação de radicais altamente reativos, como o ácido hipocloroso ( $\mathrm{HClO})$, a partir de $\mathrm{H}_{2} \mathrm{O}_{2}$ e íons halogênios $\left(\mathrm{Cl}^{\circ}, \mathrm{I}, \mathrm{Br}\right)$ que tem importante papel nos mecanismos de morte intracelular de bactérias, possivelmente através da halogenação protéica (Bogliolo, 1981). Hampton et al. (1996) reafirmaram o importante papel das enzimas superóxido dismutase e mieloperoxidase no processo oxidativo de morte de Staphylococcus aureus dentro dos neutrófilos, sendo que a primeira atua em conjunto com uma via dependente de mieloperoxidase. Segundo estes autores, a superóxido dismutase contribuiu em $25 \%$ na destruição desta bactéria e a mieloperoxidase em 33 a 40\%. Cooray e Bjorck (1995) estudaram os efeitos bactericidas do sistema mieloperoxidase/peróxido/haleto sobre bactérias patogênicas causadoras de mastite, mostrando que este sistema é extremamente efetivo contra Staphylococcus aureus, Streptococcus agalactiae, Streptococcus dysgalactiae, Streptococcus uberis e Escherichia coli.

$\mathrm{O}$ primeiro relato de deficiência de MPO foi feito em humanos por Grignaschi et al. (1963), descrevendo a deficiência completa da enzima em um paciente. Diversos autores (Cech et al., 1979; Edwards et al., 1988) observaram diminuição da capacidade bactericida de leucócitos polimorfonucleares (PMNs) de indivíduos com a deficiência de MPO, contra microrganismos como Staphylococcus aureus e Candida albicans. Nauseef (1990) descreveu que a deficiência de MPO é um defeito genético relativamente comum, ocorrendo na freqüência de 1:2000-4000 na população humana. Kitahara et al. (1979) e Parry et al. (1981), determinando a atividade de MPO numa população de pacientes de um hospital, encontraram prevalência da deficiência de MPO em neutrófilos entre $1: 2100$ e 1:4000. A MPO humana é uma proteína dimérica com duas subunidades, uma pesada com $59 \mathrm{KDa}$ e a outra leve com 13.5 KDa (Johnson e Nauseef, 1991), sendo sintetizada primariamente como uma proteína simples de $80 \mathrm{KDa}$ (Nauseef, 1986), que após a tradução sofre um processamento proteolítico, para então ser armazenada nos lisossomos neutrofílicos, durante o estágio promielocítico do desenvolvimento mielóide (Lubbert et al., 1991).
Nauseef et al. (1983) descreveram que indivíduos com deficiência hereditária de MPO não apresentavam evidências enzimáticas, imunoquímicas ou espectrofotométricas da presença de MPO ativa, verificando a existência de uma proteína imunoquimicamente relacionada à MPO, com o mesmo peso molecular de $90 \mathrm{KDa}$ e tamanho do precursor biossintético para a MPO. Com base nestes resultados, sugeriu-se que a deficiência de MPO poderia estar relacionada a um defeito no processamento pós-traducional de um precursor da MPO (Nauseef et al., 1983). Em outro estudo, Tobler et al. (1989) descreveram um quadro de deficiência completa de MPO em um indivíduo que não apresentava evidência da presença deste precursor relacionado com a MPO, sugerindo que a deficiência de MPO pode, também, ser pré-traducional. Nauseef et al. (1994) e Kizaki et al. (1994) descreveram a base genética da deficiência de MPO em humanos como sendo devida a uma mutação, causada pela substituição da base timina por citosina no "exon" 10 no nucleotídeo 8089. Analisando a mieloperoxidase bovina, Cooray et al. (1993) identificaram três diferentes formas de MPO (I, II e III) que apresentaram grande similaridade entre si, mas importantes diferenças quanto à sua atividade. Alguns fatos que sugerem a deficiência de MPO também possa acometer os bovinos: a) a deficiência de MPO descrita no homem leva a infecções recorrentes causadas por Staphylococcus aureus (Edwards et al., 1988), quadro este também muito freqüente nas infecções da glândula mamária dos bovinos; b) a deficiência de MPO pode estar associada a infecções recorrentes causadas, principalmente, por bactérias catalase positivas (Robbins et al., 1991); c) as infecções na glândula mamária dos bovinos causadas por Streptococcus agalactiae, um agente contagioso semelhante ao Staphylococcus aureus e catalase negativo, não apresentam, caracteristicamente, um quadro de infecções recorrentes (Fox e Gay, 1993); d) a raça Holandesa é altamente consangüínea, o que proporciona a disseminação de doenças ligadas a herança genética, tal como a deficiência de MPO e o BLAD ("Bovine leukocyte adhesion deficiency"); e) similaridade na seqüência de aminoácidos entre a MPO bovina e a humana.

As vitaminas antioxidantes - vitamina $E$, vitamina $C$ e o b-caroteno - têm sido apontadas como capazes de aumentar a resposta imune em várias espécies (Roth e Kaeberle, 1985). A evidência para a função antioxidante destas substâncias baseia-se na propriedade de diminuir a oxidação de membranas, enzimas, polissacarídeos, ácidos nucléicos e outras macromoléculas, causadas primariamente pela produção de radicais livres provenientes do metabolismo celular (Bendich, 1993; Nockels, 1988; Roeder, 1995). Já os ruminantes são totalmente dependentes da síntese endógena de ácido ascórbico para os seus requerimentos. Desta forma, quaisquer situações em que ocorra diminuição da disponibilidade de precursores para a síntese desta vitamina - glicose e galactose - pode haver produção insuficiente da mesma para a demanda do animal (Macleod et al., 1996). Vacas com elevada produção de leite podem estar predispostas a deficiência marginal de vitamina $C$, em virtude dos requerimentos de glicose e galactose para a síntese de leite na glândula mamária (Macleod et al., 1996). A vitamina C apresenta efeitos estimulatórios sobre a atividade fagocitária de leucócitos, sobre a função do sistema reticuloendotelial e sobre a síntese de anticorpos. No homem, Bolscher et al. (1984) demonstraram que $\mathrm{o}$ ácido ascórbico estimula a atividade de MPO in vitro; aumentando a produção de ácido hipocloroso ( $\left.\mathrm{HClO}^{-}\right)$e também alterando o pH ótimo para a atuação da MPO. Nesta rea- 
ção, grandes quantidades de $\mathrm{O}_{2} \cdot$ são produzidas, ocorrendo presença de formas inativas da MPO (II e III), o que leva à diminuição da síntese de ácido hipocloroso (Marquez et al., 1990). $\mathrm{Na}$ presença de ácido ascórbico as formas inativas são reconvertidas na forma ativa da MPO (I), o que causa aumento na formação de ácido hipocloroso e, desta forma, aumenta a atividade bactericida da MPO (Marquez e Dunford, 1990).

Desta forma, o presente trabalho teve por objetivo avaliar a correlação entre os níveis plasmáticos de ácido ascórbico e sua correlação com a atividade mieloperoxidase neutrofílica em vacas leiteiras da raça Holandesa.

\section{Material e métodos}

Foram coletadas 100 amostras de sangue de vacas da raça Holandesa uniformemente distribuídas em diferentes estágios de lactação e provenientes de três rebanhos leiteiros comerciais, localizados nos municípios de Caçapava-SP (Fazenda A), Tietê-SP (Fazenda B) e Piracicaba-SP (Fazenda C). Imediatamente após a coleta do sangue, preparava-se a extensão sangüínea, processando-as imediatamente segundo o método de Kaplow (Kaplow, 1965), que é considerada uma metodologia sensível, rápida e altamente relacionada com a atividade da MPO (Cramer et al., 1982). Para tal, foi utilizada benzidina purificada como composto indicador, em uma solução corante simples e estável que, quando oxidada, precipita-se em cristais ou grânulos marrons ou azuis (Kaplow, 1965). Os resultados são expressos na forma de escore para MPO, que é determinado pela contagem de 100 neutrófilos consecutivos, graduando-os de 0 a 4 + (cruzes), segundo a intensidade de coloração destes grânulos. $O$ ácido ascórbico foi determinado através da técnica de HPLC (High Performance Liquid Chromatography) segundo metodologia descrita por Waine e Burton (1989).

Os resultados foram analisados através do programa computacional "Statistical Analysis System" (SAS, 1985), sendo anteriormente verificada a normalidade dos resíduos pelo Teste de SHAPIRO-WILK (PROC UNIVARIATE) e as variâncias comparadas pelo Teste do qui-quadrado (Comando SPEC do PROC REG). Os dados (variável dependente) que não atenderam a estas premissas foram submetidos à transformação logarítmica $(\log X+1)$ ou pela raiz quadrada [RQ $(X+1 / 2)]$.

\section{Resultados e discussão}

A seguir são apresentados, nas Tabelas 1, 2, e 3, os resultados das médias da concentração plasmática de ácido ascórbico e o escore da atividade mieloperoxidase, de acordo com a distribuição em função da fase de lactação, número de lactações e fazendas.

Tabela 1: Concentração plasmática de ácido ascórbico e escore da mieloperoxidase (MPO) de vacas da raça Holandesa, em função do número de lactações

$\begin{array}{lcc}\text { Número de lactações } & \begin{array}{c}\text { Concentração } \\ \text { plasmática de ácido } \\ \text { ascórbico (mg/L) }\end{array} & \text { Escore de MPO } \\ \text { Multíparas } & 2.63 & 1.18 .60 \\ \text { Primíparas } & 2.59 & 95.06 \\ \text { Coeficiente de variação } & 35.97 & 78.24\end{array}$

*De acordo com o método de Kaplow.
Tabela 2: Concentração plasmática de ácido ascórbico e escore da mieloperoxidase (MPO) de vacas da raça Holandesa, em função da propriedade.

\begin{tabular}{|ccc|}
$\begin{array}{c}\text { Fazenda } \\
\text { plasmática de ácido } \\
\text { ascórbico (mg/L) }\end{array}$ & \\
\hline A & $2.73^{\mathrm{a}}$ & $83.20^{\mathrm{b}}$ \\
B & $2.85^{\mathrm{a}}$ & $48.72^{\mathrm{C}}$ \\
C & $2.15^{\mathrm{b}}$ & $221.14^{\mathrm{a}}$ \\
Coeficiente de variação & 35.97 & 78.24
\end{tabular}

Obs.: Valores com letras diferentes na mesma coluna, diferem significativamente $(P<0.05)$.

* De acordo com o método de Kaplow.

Tabela 3: Concentração plasmática de ácido ascórbico e escore da mieloperoxidase (MPO) de vacas da raça Holandesa, em função da fase de lactação.

\begin{tabular}{|c|c|c|}
\hline Fase da lactação & $\begin{array}{l}\text { Concentracão } \\
\text { plasmática de ácido } \\
\text { ascórbico }(\mathrm{mg} / \mathrm{L})\end{array}$ & Escore de MPO \\
\hline \multicolumn{3}{|l|}{ Geral } \\
\hline Seca & 2.66 & 145.58 \\
\hline $1-4$ semanas & 2.64 & 121.17 \\
\hline $5-8$ semanas & 2.60 & 97.68 \\
\hline $9-20$ semanas & 2.39 & 123.35 \\
\hline 21 - 40 semanas & 2.65 & 58.80 \\
\hline Coeficiente de variação & 35.97 & 78.24 \\
\hline \multicolumn{3}{|l|}{ Primíparas } \\
\hline Seca & 2.62 & 135.50 \\
\hline $1-4$ semanas & 2.31 & 99.33 \\
\hline $5-8$ semanas & 2.74 & 78.83 \\
\hline $9-20$ semanas & 2.27 & 93.80 \\
\hline $21-40$ semanas & 2.55 & 61.71 \\
\hline Coeficiente de variação & 35.97 & 78.24 \\
\hline \multicolumn{3}{|l|}{ Multiparas } \\
\hline Seca & 2.69 & 152.91 \\
\hline 1 - 4 semanas & 2.78 & 128.88 \\
\hline $5-8$ semanas & 2.54 & 104.26 \\
\hline $9-20$ semanas & 2.44 & 133.20 \\
\hline 21 - 40 semanas & 2.72 & 56.25 \\
\hline Coeficiente de variação & 35.97 & 78.24 \\
\hline
\end{tabular}

*De acordo com o método de Kaplow.

Foi feita a análise estatística para avaliar a associação entre a concentração plasmática de ácido ascórbico e escore de MPO. Foram analisados, também, os efeitos das variáveis número de lactação, estágio de lactação e rebanho sobre esta associação, através do teste de comparação de retas. 
Houve efeito significativo $(P=0.0101)$ da associação entre ácido ascórbico e mieloperoxidase, de acordo com a equação geral. Observou-se, ainda, que a variável número de lactações influiu significativamente nesta associação, conforme as equações abaixo:

Equação Geral:

$$
M P O=181,30-28,68 \text { (Aas) }\left(R^{2}=0.0906, P=0.0039\right)
$$

Equação por Número de Lactação:

$$
\text { Multíparas: } \mathrm{MPO}=161,82+18,08 \text { (Aas) }\left(R^{2}=0.0428, P=0.1096\right)
$$$$
\text { Primíparas: } \mathrm{MPO}=276,91+72,98 \text { (Aas) }\left(R^{2}=0.3789, P=0.004\right)
$$

Onde MPO= escore de MPO, de acordo com o método de Kaplow, e Aas = concentração plasmática de ácido ascórbico em $\mathrm{mg} / \mathrm{L}$.

Analisando-se os resultados de concentração de ácido ascórbico e escore de mieloperoxidase, verificou-se que o último apresentou alto coeficiente de variação. Tal fato pode ser atribuído ao comportamento normal desta variável na espécie bovina ou em virtude da aplicabilidade da técnica para a espécie bovina. Considerando os dados obtidos com o uso desta técnica para a avaliação da atividade enzimática da MPO, verificou-se que não houve efeito da fase de lactação e do número de lactações sobre a concentração de ácido ascórbico e escore de MPO. Entretanto, foi observado efeito significativo da fazenda sobre as variáveis conforme a Tabela 2. Uma vez que todas as fazendas apresentavam animais

\section{Referências bibliográficas}

BENDICH, A. Physiological role of antioxidants in the immune system. Journal of Dairy Science, v. 76, n. 9, p. 2789-2794, 1993.

BOGLIOLO, L. Patologia. 3. ed., Rio de Janeiro: Guanabara Koogan, 1981. BOLSCHER, B. G. J.M., ZOUTBERG, G. R., CUPERUS, R. A., WEVER, R. Vitamin $C$ stimulates the chloronating activity of human myeloperoxidase. Biochimica et Biophysica Acta, v. 784, n. 2/3, p. 189-191, 1984.

CECH, P., PAPATHANASSIOU, A., BOREUX, G., ROTH, P., MIESCHER, P. A. Hereditary myeloperoxidase deficiency. Blood, v. 53, n. 3, p. 403-411, 1979.

COORAY, R. Use of myeloperoxidase as an indicator of mastitis in dairy cattle. Veterinary Microbiology, v. 42, n. 4, p. 317-326, 1994.

, BJORCK, L. Bactericidal activity of the bovine myeloperoxidase system against bacteria associated with mastitis. Veterinary Microbiology, v. 46, n. 4, p. 427-434, 1995.

HAKANSSON, L. Defective polymorphonuclear neutrophil function in dairy cows showing enhanced susceptibility to intramammary infections. Journal of Veterinary Medicine B, v. 42, n. 10, p. 625-632, 1995.

, PETERSSON, C. G. B., HOLMBERG, O. Isolation and purification of bovine myeloperoxidase from neutrophils granules. Veterinary Immunology and Immunopathology, v. 38, n. 3-4, p. 261-272, 1993.

CRAMER, D. R., SORANJO, M. R., COMIN, A., MIOTTI, V., PATRIARCA, P. New aproaches to the detection of myeloperoxidase deficiency. Blood, v. 60, n. 2, p. 323-327; 1982.

EDWARDS, S. W., HART, C. A., DAVIES, J. M., PATTISON, J., HUGHES, V., SILLS, J. A.. Imparied neutrophil killing in a patient with defective degranulation of myeloperoxidase. Journal of Clinical Laboratory and Immunology, v .25, n. 4, p. 201-206, 1988. de padrões e origens semelhantes, a diferença encontrada pode ser devida a fatores ambientais, em especial a nutrição do rebanho.

Considerando as condições em que foram obtidos os escores de MPO, a correlação negativa encontrada in vivo é discordante dos resultados obtidos com ensaios in vitro em humanos (Bolscher et al., 1984). Desta maneira, para bovinos, esta relação parece ser inversa daquela encontrada in vitro para humanos. Quando foi adicionado ao modelo estatístico da associação entre a concentração de ácido ascórbico e atividade enzimática de MPO, as variáveis fazenda, número de lactações, estágio de lactação e suas interações, houve efeito significativo do número de lactação sobre esta associação, de forma que nas primíparas este efeito inverso foi muito mais evidente que nas multíparas. Isto significa que a associação tem comportamento diferente para multíparas e primíparas. Entretanto, não existem dados na literatura que possam esclarecer as razões para tal diferença.

\section{Conclusões}

Sob as condições em que este trabalho se desenvolveu, foi possivel estabelecer as seguintes conclusões:

- Foi encontrada associação negativa in vivo entre atividade enzimática da MPO e o nível plasmático de ácido ascórbico.

- Houve efeito significativo do número de lactações e do rebanho sobre a associação entre atividade enzimática da MPO e o nível plasmático de ácido ascórbico.

FONSECA, L. F. L. Estudo da prevalência da mastite bovina e sua relação com práticas de manejo, higiene e terapia em fazendas produtoras de leite tipo "B" no Estado de São Paulo. Dissertação (Mestrado). ESALQ/USP, Piracicaba, São Paulo, 1992.

FOX, L. K., GAY, J. M. Contagious Mastitis. The Veterinary Clinics of North America - Food Animal Practice, v. 9, n. 3, p. 475-489, 1993.

GRIGNASCHI, V. J., SPERPERATO, A. M., ETCHEVERRY, M. J., MACARIO, A. J. Un nuevo cuadro citoquímico: negatividad espontanea de las reacciones de peroxidases, oxidases e lipido en la progenie neutrofila y en los monocytos de dos hermanos. Revista Asociación Medecina Argentina, v. 77, n. 2, p. 218 , 1963.

HAMPTON, M. B., KETTLE, A. J., WINTERBOURN, C. C. Involvement of superoxide and myeloperoxidase in oxygen-dependent killing of Staphylococcus aureus by neutrophils. Infection Immunology, v. 64, n. 9, p. 3512-3517, 1996.

INTERNATIONAL DAIRY FEDERATION. Mastitis Control, results of questionnaire 1889/A. Bulletin of the International Dairy Federation, n. 262, v.15, 1991.

JOHNSON, K. R., NAUSEEFW.M. Peroxidases. In: EVERSE, J., EVERSE, K., GRISHAM, M. Chemistry and Biology. Boca Raton: CRC Press, 1991, p. 63-82.

KAPLOW, L. S. Simplified myeloperoxidase stain using benzidine dihydrocloride. Blood, v. 26, n. 2, p. 215-218, 1965.

KITAHARA, M., SIMONIAN, Y., EYRE, H. J. Neutrophil myeloperoxidase: a simple, reproducible technique to determine activity. Journal of Laboratory Clinical Medicine, v. 93, n. 2, p. 232-237, 1979.

KIZAKI, M., MILLER, C. W., SELSTED, M. E., KOEFLER, H. P. Myeloperoxidase (MPO) gene mutation in hereditary MPO deficiency. Blood, v. 83, n. 7, p. 1935-1940, 1994.

KLEBANOFF, S. J. Peroxidases. In: EVERSE, J., EVERSE, K., GRISHAM, M. Chemistry and Biology. Boca Raton: CRC Press, 1991, p. 1-36. 
LANGENEGGER, J., COELHO, N. M., LANGENEGGER, C. H., CASTRO, R. P. Estudo da incidência da mastite bovina na bacia leiteira do Rio de Janeiro. Pesquisa Agropecuária Brasileira, v. 5, n. 3, p. 437, 1970.

LANGONI, H., PINTO, M. P., DOMINGUES, P. F. LISTONI, F. J. P. Mastite bovina subclínica, etiologia e sensibilidade bacteriana. Comunicações Científicas da Faculdade de Medicina Veterinária e Zootecnia-USP, v. 14 n. 1, p. 13, 1990.

LUBBERT, M., HERRMANN, F., KOEFFLER, H. P. Expression and regulation of myeolid-specific genes in normal and leukemic myeloid cells. Blood, v. 77, n. 5, p. 909-924, 1991.

MACLEOD, D., OZIMECK, L., KENNELLY, J.J. Supplemental vitamin C may enhance immune system in dairy cows. In: Advances in Dairy Technology - Western Dairy Seminar, 8. Alberta, 1996. Alberta, University of Alberta. 1996, p. 227-230.

MARQUEZ, L. A., DUNFORD, H. B. Reaction of compound III of myeloperoxidase with ascorbic acid. The Journal of Biological Chemistry, v. 265, n. 11, p.6074-6078, 1990.

MARQUEZ, L. A., DUNFORD, H. B., VANWART, H. Kinetic studies on the reaction of compound II of myeloperoxidase with ascorbic acid. The Journal of Biological Chemistry, v. 265, n. 10, p.5666-5670, 1990.

NAUSEEF, W. M. Myeloperoxidase byosyntesis by a human promyeolocytic leukemia cell line: insight into myeloperoxidase differentiation. Blood, v. 67, n. 4, p. 865-872.1986.

NAUSEEF, W. M. Myeloperoxidase deficiency. Hematology Pathology, v. 4, n. 4, p. 165-178, 1990.

NAUSEEF, W. M., BRIGHAM, S., COGLEY, M. N. Hereditary myeloperoxidase deficiency due to a missense mutation of arginine 569 to tryptophan. The Journal of Biological Chemistry, v. 269, n. 2, p. 1212-1216, 1994.

NAUSEEF, W. M., ROOT, R. K., MALECH, H. L. Biochemical and immunological analysis of hereditary myelopeoxidase deficiency. Journal of Clinical Investigation, v. 71, n. 5, p. 1297-1307, 1983.

NOCKELS, C. F. The role of vitamins in modulating disease resistance. Veterinary Clinics of North America: Food Animal Practice, v. 4, n. 3, p. 531-542, 1988.
PARRY, M. F., ROOT, R. K., METCALF, J. A., DELANEY, K. K., KAPLOW, L. S., RICHAR, W. J. Myeloperoxidase deficiency prevalence and clinical significance. Annals of InternalMedicine, v. 95, p. 293-301, 1981.

PHILPOT, W. N., NICKERSON, S. C. Mastitis: Counter attack. A strategy to combat mastitis. Illinois: Babson Bros. Co., 1991.

ROBBINS, S. L., COTRAN, R. S., KUMAR, V. Patologia Estruturale Funcional. 4. ed. Rio de Janeiro: Ed. Guanabara Koogan, 1991.

ROEDER, R. A. Beyond deficiency: new views of vitamins in ruminant nutrition and health: an overview. The Journal of Nutrition, v.125, n. 6S, p.1790-1791, 1995.

ROTH, J. A., KAEBERLE, M. L. In vivo efect of ascorbic acid on neutrophil function in heathy and dexametasone-treated cattle. American Journal of Veterinary Research, v. 46, n. 12, p. 24342436, 1985

SANTOS, M. V., FONSECA, L. F. L., LUCCI, C. S., HEINEMANN, M. B., RICHTZENHAIN, J., VISINTIN, J. A., GARCIA, F. Determination of myeloperoxidase activity in neutrophils from Staphylococcus aureus mastitis holstein cows. In: Annual Meeting of National Mastitis Council, 35. Tennesseee, 1996. Anais. Wisconsin, National Mastitis Council, 1996, p.180-182.

SAS INSTITUTE. SAS User Guide: Statistics. 1985 ed. Cary SAS Institute, 1985.

SEARS, P. M. Staphylococcus aureus mastitis. In: Annual Meeting National Mastitis Council, 32. Missouri, 1993. Anais. Wisconsin, National Mastits Council, 1993, p. 4-11.

TOBLER, A., SELSTED, N. E., MILLER, C. W., JOHNSON, K. R., NOVOTNY, M. J., ROVERS, G., KOEFFER, H. P. Evidence for a prettranslational defect in hereditary and acquired myeloperoxidase deficiency. Blood, v. 73, n. 7, p. 1980-1986, 1989.

WAYNE, D. D. M., BURTON, G. W. Measurement of individual antioxidants and radical-trapping acitivity. In: NIQUEL, J., QUINTANILHA, A. D., WEBER, H. CRC Handbook of Free Radical and Antioxidants in Biomedicine. Vol. III, Boca Raton: CRC Press, 1989 , p. 223-232.

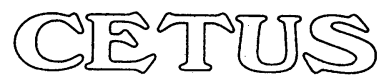

NIKON (Japão)

Distribuidor Nacional

Cetus Hospitalar Comércio e Representações Ltda.

Microscópios Biológicos e Industriais - Espectofotômetros Deonizadores - Kits para bioquímica - Contador de Células

\section{Assistência Técnica}

Rua Lopes Trovão, 75 - Benfica

20920-310 - Rio de Janeiro - RJ

Tel.: (21) 568-9345

Fax: (21) 264-3628

E-mail: cetus@abeunet.com.br 